\title{
Association between resuscitation time interval at the scene and neurological outcome after out-of- hospital cardiac arrest in two Asian cities.
}

\section{AUTHOR(S):}

Shin, Sang Do; Kitamura, Tetsuhisa; Hwang, Seung Sik; Kajino, Kentaro; Song, Kyoung Jun; Ro, Young Sun; Nishiuchi, Tatsuya; Iwami, Taku; for the Seoul-Osaka Resuscitation Study (SORS) Group

\section{CITATION:}

Shin, Sang Do ... [et al]. Association between resuscitation time interval at the scene and neurological outcome after out-of-hospital cardiac arrest in two Asian cities..

Resuscitation 2014, 85(2): 203-210

\section{ISSUE DATE:}

2014-02

URL:

http://hdl.handle.net/2433/182212

RIGHT:

(C) 2013 Elsevier Ireland Ltd. 
1 Association between resuscitationtime intervalat the scene andneurological 2 outcomeafter out-of-hospital cardiac arrest in two Asian cities

4 Sang Do Shin ${ }^{a}$, Tetsuhisa Kitamura ${ }^{\text {, }}$, SeungSikHwang ${ }^{c}$, KentaroKajino ${ }^{\text {, }}$, Kyoung Jun

5 Song $^{\mathrm{e}}$, Young Sun Ro ${ }^{\mathrm{f}}$, Tatsuya Nishiuchi ${ }^{\mathrm{g}}$, TakuIwami ${ }^{\mathrm{h}}$, for Seoul-Osaka Resuscitation

6 Study (SORS) Group

${ }^{a}$ Department of Emergency Medicine, Seoul National University College of Medicine, Seoul,

101 Daehak-Ro, Jongno-Gu, Seoul 110-744, Korea

${ }^{b}$ Division of Environmental Medicine and Population Sciences, Department of Social and

11 Environmental Medicine, Graduate School of Medicine, Osaka University, 2-5 Yamada-oka,

Suita, Osaka 565-0871, Japan

${ }^{c}$ Department of Social Medicine, Inha University, Shinheun-Dong 3 Ga, Jung-Gu, Incheon

400-712, Korea

${ }^{d}$ Department of Traumatology and Acute Critical Medicine, Osaka University Graduate

School of Medicine, 2-5 Yamada-oka, Suita, Osaka 565-0871, Japan 
$19{ }^{f}$ Department of Preventive Medicine, Seoul National University School of Public Health,

20 Seoul, 1 Kwanak-Ro Kwanak-Gu, Seoul 151-741, Korea

$21{ }^{g}$ Department of Critical Care \& Emergency Medicine, Osaka City University Graduate

Total word count of the paper: 5396 words

Total word count of the abstract: 256words

\section{Number of figures: 2}

\section{Number of tables: 5}

* Corresponding author. Phone: +81-75-753-2401; Fax:+81-75-753-2424.

TakuIwami MD PhD

32 Kyoto University Health Service

33 YoshidaHonmachi, Sakyo-ku, Kyoto 606-8501, Japan

$$
\text { E-mail address: iwamit@e-mail.jp (T. Iwami) }
$$


37 Cardiopulmonary resuscitation

38 Ambulance

39 Out-of-hospital cardiac arrest

40 Emergency medical service system

41 Scene Time Interval

42

43

44

45

46

47

48

49

50

51

52

53

54 
Background: It is unclear whether the scene time interval (STI) for cardiopulmonary resuscitation (CPR) is associated with outcomes of out-of-hospital cardiac arrest (OHCA) or not.

Methods: A retrospective analysis based on two large population-based cohortswas performed forwitnessed adult OHCA with presumed cardiac etiology from Seoul (2008-2010) and Osaka (2007-2009). The STI defined as time from wheel arrival at the scene to departure to hospital was categorized by short (less than 8 minutes), intermediate ( 8 to less than 16 minute), and long (16 min or longer) STI on the basis of sensitivity analysis. Primary outcome was good neurological outcome (cerebral performance category 1 or 2).Adjusted odds ratios (AORs) with $95 \%$ confidence intervals (CIs) were calculated to determine the association between STIs and outcomes comparing with the short STI group adjusting for potential risk factorsand interaction products.

Results: Total 7,757 patients;3,594 from Seoul and 4,163 from Osaka were finally analyzed.

There were significant differences among STI groups for most potential risk variables.

Survival to admission was higher in the intermediate STI group (35.7\%) than in the short (31.8\%) or long STI group (32.6\%), respectively ( $p=0.004)$. Survival to discharge was not 
73 group had a significantly better neurological outcome compared with the short STI group

74 (7.7\% vs. $4.6 \%$; AOR, 1.32; 95\% CI, 1.03-1.71), while the long STI (6.6\%) did not.

75 Conclusion: Data from two metropolitan cities demonstrateda positiveassociation between

76 intermediate STI from 8 to 16 minutes and good neurological outcome after OHCA.

77

78

79

80

81

82

83

84

85

86

87

88

89

90 
91

92

93

94

95

\section{Introduction}

Out-of-hospital cardiac arrest (OHCA) is a major publichealth problem around the world. ${ }^{1}$ Emergency medical service (EMS) factors have been known to be one of the major determinants for outcomes after OHCA as well as bystander performance and hospital postresuscitation care. ${ }^{2-5}$ Among EMS factors, response time interval (RTI) from call to arrival at the scene isknown to be associated with outcomes, whereas the effect ofscene time interval (STI) for cardiopulmonary resuscitation (CPR), from ambulance arrival at the scene to departure to hospital, isunclear.

Although some EMS systems like Osaka allow longer STI than others, in many Asian countries including Korea and Japan, EMS protocols do not basically allow them to stop CPR at the scene unless there is a return of spontaneous circulation (ROSC) and require scoop and run to the hospital emergency department (ED) while giving CPR during transport (Scoop and Run model). ${ }^{6-9}$ In addition, advanced life support (ALS) procedures provided by EMS personnel on the scene are very limited. These protocols are very different from those in North America, Europe, and Australia,where EMS providers continue to perform CPR until getting the ROSC or stopping CPR for death declaration on the scene (Stayand Treat model). ${ }^{10,11}$ The 2010 American Heart Association guideline has no comments regarding how long the EMS personnel stay at the scene to provide CPR or how many CPR cycles 
areessential for scene CPR. ${ }^{10,11}$

We hypothesizedthat the STI staying at the sceneto provide CPR is a key determinant for outcomes in OHCA in EMS systems because it is a very important treatment time interval after cardiac arrests.During this period, EMS personnel would provide varioustreatments at

113 the scene including CPR, defibrillation, airway management, and fluid resuscitation. ${ }^{12}$ The

114 longer STI for CPR has a benefit of providing a likelihood of more stable and higher-quality

115 CPR, while the shorter STI has a benefit of faster, more comprehensive and earlier advance

116 care in ED. In contrast, there would be a disadvantage of delayed advance care by ED in the

117 longer STI protocol,butmore unstable CPR during ambulance transport in the shorter

118 STIsystem. Bycomparing the outcomesaccording to STI for CPR, we can develop amore

119 effective scene protocol for the EMS system. Thepresent study aimed to determine the association between STI and neurological outcomeafter OHCA using two large populationbased cohorts covering two metropolitan cities in Asia.

\section{Methods}

\subsection{Study method and materials}


127 respectively. The study was approved by the institutional review boards of the study

128 hospitals(Seoul National University and Osaka University).

129

130

131

132

133

134

135

136

137

138

139

140

141

\subsection{Study setting}

This study was done in two metropolitan cities, Seoulin Korea and Osaka in Japan. The population size was 9.9 million in Seoul (2009) and 8.8 million in Osaka (2010). Population density was 16,356 per $\mathrm{km}^{2}$ in Seoul and 4,642 per $\mathrm{km}^{2}$ in Osaka.

The EMS level is intermediate in both areas where the highest-qualifiedemergency medical technicians can give CPR with automatic external defibrillation (AED), perform advanced airway management, and insert intravenous fluid. EMS providers should not stop CPR unless there is a ROSCon the scene. In Seoul, EMTs are encouraged to "scoop and run" to a hospital emergency departmentwhile giving CPR during ambulance transport as soon as possible after giving one cycle of CPR followed by automatic external defibrillation.More than one cycle of CPR and rhythm analysis with/without shock at the scene, intravenous fluid resuscitation, advanced airway management, and drug infusionare not obligatory but optional in this system. EMS providers canchoose their option according to families' attitude, patient's condition, self-confidence level for procedures, and expected transport time to ED. They give continuous CPR during ambulance transport. ${ }^{13}$ In Osaka, EMTs are usually encouraged tostay around 10 minutes for interventionsincludingthree to four cycles of CPRwith rhythm analysis 
145 every 2 minutes, intravenous fluid resuscitation, advanced airway management, and drug

146 infusion. $^{14}$ The emergency life-saving technician (ELST) systems in Osaka were started in

147 1991;specially-trained ELSTs began to use endotracheal intubation in July 2004, and

148 administered adrenaline since July 2006, while the first-degree EMTs in Seoul can opt for the

149 airway managementfrom among Bag-Valve-Mask ventilation, supraglottic airway, and

150 endotracheal intubation according to their preference. A system quality program was initiated

151 in 1998 in the Osaka through Osaka Utstein Project, while the quality control was rare in

Seoul until 2011.

The number of ambulance crew per ambulance isthree in both areas;first-degree EMT

(intermediate EMT), second-degree EMT (basic EMT), and driver (first responder level) in

Seoul, andthree EMSs with at least one ELST (intermediate EMT)in Osaka. The first-degree

EMTs in Seoul are usually graduatesof EMT colleges with three- to four-year courses. In

157 Osaka, there are two processes to qualify as an ELST. ${ }^{14}$ The first is through the specialized education system in the fire department. The second way is through the education system in the EMT school and college.

There are 114 ambulance unitsavailable for service coverage of 24-hour/ 365 days in 
163 (level 2). Level 1 and 2 EDs are covered by emergency physicians for 24/365 services and

164 received approximately 80\% of OHCA.In Osaka, there are 13 critical care medical centers

165 (level 1) and 258 emergency centers (level 2). Level 1 ED can be used for very high

166 criticality such as OHCA, severe trauma, and respiratory failure, and has received approximately $20 \%$ of OHCA, every yearwhile level 2 EDs are used for moderate emergency conditions. The classification of ED levels differ by areas. The level 1 and some of level 2 hospitals in both cities usually provide therapeutic hypothermia and cardiac interventions (percutaneous coronary intervention and coronary bypass surgery).

\subsection{Study population}

172 Study population was EMS-treated and bystander-witnessed adult (18 years and older) OHCA with presumed cardiac etiology. The study period was from Jan. 2008 to Dec. 2010 for Seoul and from Jan. 2007 to Dec. 2009 for Osaka. OHCA with non-cardiac etiology, not treated cases, and patients less than 18 years were excluded.

\subsection{Data sources} hospital medical record review in Seoul. Utstein factors including age, sex, location(public 
181 definition according to the Utstein data report form. In Osaka, first documented ECG was

182 examined at the scene, while that in Seoul was examined for some patients at the scene and

183 for most patients at the ED.Public accessdefibrillation was performed for several cases in

184 Osaka while there was no case in Seoul becausethe PAD program started in 2010 in that city.

185 Dataset details were reported in previous studies. ${ }^{5,8,13-16}$

The elapsed time intervals such as response time interval (RTI) from call to wheel arrival

at scene, scene time interval (STI) from wheel arrival to departure to ED, andtransportation

time interval (TTI) from departure at thesceneto arrival at EDwere standardized and

measured in both areas.

\subsection{Outcome measure}

191

Primary outcome was good neurological outcome at hospital discharge (Seoul) and

atonemonth after event (Osaka), which was defined as cerebral performance category 1 or2.

The secondary outcome was survival to discharge (Seoul) or onemonth survival (Osaka), and survival to admission.

Outcomes were collected by EMS providers onemonth after transporting patients to EDs in Osaka via telephone or fax contact to hospital. In Seoul, hospital medical record review was performed after hospital discharge. Medical record review was done by medical record experts employed at Korean Centers for Disease Controls and Prevention.Information on 
199 neurological outcome was unavailable for six patients $(0.2 \%)$ in Seoul and five cases $(0.1 \%)$

200 in Osaka.

201

\subsection{Main exposure variables}

202

We categorized the STI according to the sensitivity analysis. Restrictive cubic spline

logistic analysis with five nodes was used to determine the cut-off value for categorizing STI

subgroups. The analysis used two variables (scene time intervals and good neurological

was categorized with short STI, intermediate STI, and long STI using two cut-values.

\subsection{Statistical analysis} mean withstandard deviation or median with the interquartile range. Categorical variables are measured by number and percent. Descriptive analysis used the Student $t$-test for continuous variables and chi-square test for categorical variables. ANOVA test was used for the 
217 STI group (reference=short STI) using multivariable logistic regression models. Potential risk

218 factors were age, sex, RTI, TTI, place, bystander CPR, primary ECG, and prehospital

219 defibrillation by layperson or EMS providers. These factors have been known to be

220 associated with outcomes in many previous studies. Study city (Seoul and Osaka) would also

221 be a potential risk although we adjusted for many relevant variables in the model. First, we

222 tested interactions between STI and city on the outcomes because thetwo cities significantly

223 showed the different STI distribution. After testing forinteractions, the city level was also

224 incorporated into the final model because there was no significant interaction.

We assessed the interaction between main exposure (STI) and other potential risk factors

226 for main outcome using the chunk test and followed by backward elimination process for full

227 model which included main exposure, potential risks, and all potential interaction products.

228 We dropped out the interaction products according to the order of size of $p$-value $>0.01$ using

229 the analysis of maximum likelihood estimates in the model. The interaction term with the

biggest $p$-value was removed from the model and also assessed the remaining interaction terms. This backward elimination processes were repeated to get final model where all potential confounders and significant interactions remained. If there was any significant interaction with $p$-value $<0.01$ between main exposure and other potential risks, we calculate 
235 plus effect modifiers on outcomes in each subgroup.

\section{Results}

Total 3,594 patients were enrolled from Seoul among 10,122 from 2008 to 2010

excluding EMS-not treated $(n=1,511)$, less than 18 years $(n=232)$, unwitnessed $(n=4,279)$, and non-cardiac etiology $(n=506)$. A total of 4,163 patients among 21,032 patients from 2007 to

2009 in Osaka were included, excluding no-treated EMS $(n=1,645)$, less than 18 years old performed, bystander and EMS defibrillation, ventricular fibrillation/tachycardia was significantly higher in Osaka than Seoul. Pre-hospitalROSC, survival to admission and survival to discharge (or one-month survival), and good neurological outcome weresignificantly better in Osaka than Seoul (Table 1).

We found the three peaks in the probability of survival in the restricted cubic spline curve; the first peak wasdownward around $5 \mathrm{~min}$ between 0 to $7 \mathrm{~min}$, when the portion of intact neurological outcome was the lowest, the second upward around $12 \mathrm{~min}$ between 8 to $15 \mathrm{~min}$, 
253 values for categorizing the STI using $8 \mathrm{~min}$ and $16 \mathrm{~min}$,respectively, responding to average

254 value of good neurological outcome (6.4\%) (Fig. 2).The STI was categorized with three

255 groups; short STI for 0 to $7 \mathrm{~min}$, intermediate STI for 8 to $15 \mathrm{~min}$, long STI for $16 \mathrm{~min}$, and

256 longer.

257

There were significant differences among STI groups for city, gender, age group, place of arrest, bystander CPR, bystander defibrillation, defibrillation by EMS provider, primary ECG rhythms, RTI, but not TTI(Table 2). Survival to admissionor to discharge and good neurological outcome was highest in the intermediate STI group (35.7\%, 13.7\%, 7.7\%) compared to the short(31.8\%, 13.1\%, 4.6\%)or the long STI group $(32.6 \%, 11.5 \%, 6.6 \%)$, in the intermediate STI group (3.6\%) while that in Osaka in the short STI group (11.4\%). The city level and STI group for prehospital ROSC ( $\mathrm{p}=0.711)$, survival to admission $(p=0.724)$, survival to discharge ( $p=0.046)$, and good neurological outcome $(p=0.265)$. 
$27195 \%$ CI;0.68-1.26), compared with short STI. Survival to discharge was significantly lower in

272 long STI than in short STI (AOR=0.64, 95\% CI;0.52-0.80), but not different in intermediate STI $(A O R=0.88,95 \% \mathrm{CI}=0.74-1.05)$. Prehospital ROSC was significantly greater both in the intermediate STI group (AOR=2.78, 95\% CI;2.18-3.54) and long STI group (AOR=4.23, 95\% CI;3.26-5.49) compared to the short STI group. Survival to admission was not significantly associated with the STI groups. djusted ORs with 95\% CIs for good neurological outcome was significantly higher in the intermediate STI group (AOR 1.40, 95\% CI=1.08-1.82), but not different in long STI (AOR 1.01, 95\% CI=0.74-1.39). However, either intermediate orlong STI group w as associated with worse neurological outcomeamong patients who received bystander defibrillation; AOR 0.20 , 95\% CI (0.05-0.92) in intermediate STI and AOR 0.09, 95\% CI (0.02-0.44) in long STI group. Both intermediate and long STI were significantly associated with higher prehospital ROSC among patients who did not receive bystande 
ediate STI group (AOR 0.33, 95\% CI 0.09-1.21) among patients who received bystan der defibrillation. There was no effect modifier on survival to discharge and to admission.

\section{Discussion}

By use of data from two large population-based registriesof OHCA in metropolitan cities, we successfully demonstratedthe benefit of EMS activities during intermediate STI from 8 to 16 min forimproving neurological outcome after OHCA.

In Osaka,the OHCA registry was startedin 1998 based on the international guideline for reporting outcomes after OHCA (Utstein Osaka Project). ${ }^{14,15}$ In Seoul,a similar OHCA registry was started in $2006,{ }^{13,16}$ and both registriesremain on-going. Recently, in Asia, the attemptto developan international registry of OHCA based on the Utstein style across countries is proceeding. ${ }^{17}$ This international collaboration makes it possible to gatherthe large number of standardized data from two largeareassuch as this with different EMS systems,in order to evaluate these important issues.

These data suggest that EMS personnel should perform CPR on the scene at least 8 to 16 minutes before transport to the ED even in the intermediate EMS level where the ALS measurements are limited. The best STI should depend on many factors including EMS levels, the ALS measures the EMS personnel can provide, hospital levels, and EMS response times. 
307 There are many differences in the EMS systems between Osaka and Seoul. Less than 10\% of

308 Seoul EMS providers inserted advanced airways, whereas Osaka providers usually selected

309 more than $70 \%$ for advanced airways. This discrepancy wouldarise from the different CPR

310 protocolsemployed in each area. Seoul EMS encourages EMS providers to give CPR

311 with/without shock delivery if indicated and to run as soon as possible, while Osaka EMSs

312 encourage providers to run after providing ALS measures including airway, fluid

313 resuscitation, and CPR with/without shock delivery. As a result, Seoul showed very short STI

314 (mean 6.8, standard deviation4.2 min) while Osaka had a longer STI (mean 14.5, standard

315 deviation $6.5 \mathrm{~min}$ ). The longer STI in Osaka might be one of the reasons for the better

316 outcome of Osaka than Seoul observed in this study. Improving neurological outcome in the

317 intermediate STI group shown in this study covering two different areas suggests that this

318 resultshowing the importance of EMS activities on the scene during early phase of cardiac

arrest is robust across different EMS systems.

We assume that the lower quality of CPR during ambulance transport might be one of the

possible explanations for thepoorest outcome in the short STI group. It is well known that the

quality of CPR is the key to improve outcomes after cardiac arrests. ${ }^{18,19} \mathrm{CPR}$ during 
325 result in lower chance of ROSC in the early phase of cardiac arrest and worse neurologic

326 outcome. Longer stay gives a chance to perform higher quality CPR and critical procedures

327 duringthe very critical time window compared to earlier departure for ED.

In intermediate service level EMS, hospital level performance is strongly associated with

survival. ${ }^{21,22}$ Earlier intervention of advance life support measures would be beneficial in

those who do not respond to treatments by EMS providers. In this study, we found the

positive association between longer STI and prehospital ROSC as well as good neurological

outcome. Longer CPR at the scene would be beneficial for achieving ROSC at the scene as

well as final better neurological outcome. Although hospital care options were not adjusted in

this study, a significant association between longer STI and ROSC before hospital arrival

supportsthe idea that the longer stay and giving CPR are very important determinant in the

EMS “Scoop and Run model.”

We found that bystander defibrillation was a strong effect modifier of STI on good

neurological outcome and preshopital ROSC. If the patient did not receive bystander defibrillation, intermediate STI was associated with good nuerological outcomewhile both the intermediate and logh STI groupswere associated with worse outcome among patients who received bystander defibrillation. Thses findings would be supported by the three phase model of CPR (1. electrical phase, from the time of cardiac arrest to approximately 4 minutes 
343 following the arrest; 2. circulatory phase, from approximately 4 to 10 minutes after cardiac

344 arrest; 3. metabolic phase, extending beyond approximately 10 minutes after cardiac arrest). ${ }^{23}$

345 When OHCA patient does not receive any defibrillation by bystanders, the intermediate STI

346 which means longer CPR time by the EMS personnel at the scene would have benefit

347 because the patient is not under the electrical phase. On the other hand,among patients who

348 has shosckable rhythm and consequently receive the bystander defibrillation in the early

349 electrical phase, the benefit of longer STI would be disappeared. These findings can be

considered for developing CPR protocol in scoop and run EMS model.

Different from our "Scoop and Run" model, which has been argued as better for safety as

well as quality of $\mathrm{CPR}^{24,25}$ and widely introduced in many Asian countries, most systems in

North America and European EMS allow EMS providers to go on CPR until archiving ROSC

or CPR termination under medical oversight unless there is a response. The best timing for

stopping CPR on the scene and starting transport to the ED should be different in a "Stayand

Treat Model" where EMS providers can provide more ALS measures. However, there still

should be additional advanced treatments that can only be provided after hospital

arrival;moreover, some studies showed the effectiveness of these treatments. ${ }^{26,27}$ In addition, 
361 of resuscitation might be too short and thatefforts to systematically increase the duration of

362 resuscitation could improve survival after cardiac arrests. ${ }^{28}$ The appropriate STI should be

363 also discussed in this type of EMS system.

364 Although we found STIeffective for good neurological outcome after OHCAin this study, the appropriate CPR protocolduring this phase is unclear. Theoretically, the short STI from 0 to 7 min would give a chance for four cycles of CPR and rhythm analysis, and median STI from 8 to 15 min would give four more cycles of CPR and rhythm analysis based on the current CPR guideline. ${ }^{10} \mathrm{STI}$ of 8 to $16 \mathrm{~min}$ is not tooshort to allowEMS resuscitation includingCPR, shocks, and some advanced treatments at the scene for OHCA victims. In Arizona, more simplified and compression highlighted CPR protocol was encouraged for EMS providers to provide continuous high-quality CPR at least 8 min without any procedures to interrupt the CPR continuity,and its effectiveness was reported. ${ }^{29,30} \mathrm{~A}$ CPR protocol for EMS providers to provide high-quality $\mathrm{CPR}$ during resuscitation on the scene should also be established.

This study has some limitations. First, it is not a randomized controlled trial on the effectsof scene time intervals on OHCA outcomes. Although we adjusted for potential risk confounders, it was limited. The second limitation derives from the study setting. This study was conducted at anintermediate service level at which EMS providers should run to ED 
379 while giving CPR during ambulance transport unless there is any ROSC. The study results

380 have limitation to be generalized. The third drawback is that we did not directly measure the

381 treatment options during the scene stay. We just regarded the scene time interval as a treatment time including giving CPR, airway management, shock delivery, and intravenous

fluid resuscitation. However, the scene time also include the time usage for failed intubation and reattempts, technical errors, not to mention the time searching for the patient. Therefore, the scene time interval does not always mean actualtreatment time at the scene. Fourth, we did not measure the CPR quality directly, for example, compression depth, CPR fraction,

CPR interruption for any procedure. These items are really of crucial importance to improve the outcomes of OHCA. A final limitation should be pointed out. Post-resuscitation care procedures including therapeutic hypothermia and cardiac intervention have been proved very critical interventions for survived patients after OHCA for good neurologic outcome as well as survival to discharge. ${ }^{31}$ The two cities had very different hospital care systems. Seoul had one level-1 and 27 level-2 EDs and Osaka had 13 level-1 critical care centers. However, service level, capacity, procedure, and protocol in two cities were neither standardized and nor measured in this study. Therefore, we could not adjust for the hospital level, capacity, and post-resuscitation care procedures. These incomplete adjustments might cause bias for study results. 


\section{Conclusion}

399 Based on a large population-based cohort covering two metropolitan cities, we found 400 apositive association between intermediate STI from 8 to 16 minutes and good neurological 401 outcome after OHCA. A better EMS resuscitation protocolshould be developed considering 402 this important resuscitation time period on the scene.

There are no conflicts of interest to declare.

406

407 Role of funding source

408 The study was funded by the Pfizer Health Research Foundation and the Korean Centers for 409 Disease Control and Prevention.

\section{$411 \quad$ References}

412 1. Nichol G, Thomas E, Callaway CW, et al. Resuscitation Outcomes Consortium

413 Investigators. Regional variation in out-of-hospital cardiac arrest incidence and outcome. 
415 2. Stiell IG, Wells GA, Field B, et al. Ontario Prehospital Advanced Life Support Study

416 Group. Advanced cardiac life support in out-of-hospital cardiac arrest. N Engl J Med $417 \quad 2004 ; 351: 647-56$.

418 3. Spaite DW, Hanlon T, Criss EA, et al. Prehospital cardiac arrest: the impact of

419 witnessed collapse and bystander CPR in a metropolitan EMS system with short response 420 times. Ann Emerg Med 1990;19:1264-9.

421 4. Stiell IG, Wells GA, DeMaio VJ, et al. Modifiable factors associated with improved 422 cardiac arrest survival in a multicenter basic life support/defibrillation system: OPALS Study 423 Phase I results. Ontario Prehospital Advanced Life Support. Ann Emerg Med 1999;33:44-50.

$4245 . \quad$ Bernard SA, Gray TW, Buist MD, et al. Treatment of comatose survivors of out-of425 hospital cardiac arrest with induced hypothermia. N Engl J Med 2002;346:557-63.

426 6. Ahn KO, Shin SD, Suh GJ, et al. Epidemiology and outcomes from non-traumatic 427 out-of-hospital cardiac arrest in Korea: A nationwide observational study. Resuscitation $428 \quad 2010 ; 81: 974-81$.

$4297 . \quad$ Ma MH, Chiang WC, Ko PC, et al. Outcomes from out-of-hospital cardiac arrest in 430 Metropolitan Taipei: does an advanced life support service make a difference? Resuscitation 2007;74:461-9. 
433 Working Group for the All-Japan Utstein Registry of the Fire and Disaster Management

434 Agency. Nationwide public-access defibrillation in Japan. N Engl J Med 2010;362:994-1004.

435 9. Ong ME, Ng FS, Anushia P, et al. Comparison of chest compression only and

436 standard cardiopulmonary resuscitation for out-of-hospital cardiac arrest in Singapore.

437 Resuscitation 2008;78:119-26.

438 10. Hazinski MF, Nolan JP, Billi JE, et al. Part 1: Executive summary: 2010 International

439 Consensus on Cardiopulmonary Resuscitation and Emergency Cardiovascular Care Science

440 With Treatment Recommendations. Circulation 2010;122:S250-75.

441 11. Sandroni C, Nolan J; European Resuscitation Council. ERC 2010 guidelines for adult 442 and pediatric resuscitation: summary of major changes. Minerva Anestesiol. 2011;77:220-6.

443 12. Shin SD, Hock Ong ME, Tanaka H, et al. Comparison of Emergency Medical 444 Services Systems Across Pan-Asian Countries: A Web-based Survey. Prehosp Emerg Care 2012;16:477-496.

446 13. Shin SD, Ahn KO, Song KJ, Park CB, Lee EJ. Out-of-hospital airway management 447 and cardiac arrest outcomes: a propensity score matched analysis. Resuscitation 2012;83:313-

449 14. Kajino K, Iwami T, Kitamura T, et al. Comparison of supraglottic airway versus endotracheal intubation for the pre-hospital treatment of out-of-hospital cardiac arrest. 
$451 \quad$ CritCare 2011;15:R236.

452 15. Iwami T, Kawamura T, Hiraide A, et al. Effectiveness of bystander-initiated cardiac-

453 only resuscitation for patients with out-of-hospital cardiac arrest. Circulation 2007;116:2900-

4547.

455 16. Park CB, Shin SD, Suh GJ, et al. Pediatric out-of-hospital cardiac arrest in Korea: A

456 nationwide population-based study. Resuscitation 2010;81:512-7.

457 17. Ong ME, Shin SD, Tanaka H, et al. Pan-Asian Resuscitation Outcomes Study

458 (PAROS): rationale, methodology, and implementation. Acad Emerg Med 2011;18:890-7.

459 18. Olasveengen TM, Wik L, Steen PA. Quality of cardiopulmonary resuscitation before

460 and during transport in out-of-hospital cardiac arrest. Resuscitation 2008;76:185-90.

461 19. Krarup NH, Terkelsen CJ, Johnsen SP, et al. Quality of cardiopulmonary 462 resuscitation in out-of-hospital cardiac arrest is hampered by interruptions in chest 463 compressions - A nationwide prospective feasibility study. Resuscitation 2011;82:263-9.

464 20. Wang HC, Chiang WC, Chen SY, et al. Video-recording and time-motion analyses of 465 manual versus mechanical cardiopulmonary resuscitation during ambulance transport.

466 Resuscitation 2007;74:453-60.

467 21. Shin SD, Suh GJ, Ahn KO, Song KJ. Cardiopulmonary resuscitation outcome of outof-hospital cardiac arrest in low-volume versus high-volume emergency departments: An 
469 observational study and propensity score matching analysis. Resuscitation 2011;82:32-9.

470 22. Kajino K, Iwami T, Daya M, et al. Impact of transport to critical care medical centers

471 on outcomes after out-of-hospital cardiac arrest. Resuscitation 2010;81:549-54.

472 23. Weisfeldt ML, Becker LB.Resuscitation after cardiac arrest: a 3-phase time-sensitive

model. JAMA2002;288:3035-8.

474 24. Kahn CA, Pirrallo RG, Kuhn EM. Characteristics of fatal ambulance crashes in the

475 United States: an 11-year retrospective analysis. Prehosp Emerg Care 2001;5:261-9.

25. Sasson C, Hegg AJ, Macy M, Park A, Kellermann A, McNally B; CARES

Surveillance Group. Prehospital Termination of Resuscitation in Cases of Refractory Out-of-

Hospital Cardiac Arrest. JAMA 2008;300:1432-8.

26. Nagao K, Kikushima K, Watanabe K, et al.Early induction of hypothermia during

cardiac arrest improves neurological outcomes in patients with out-of-hospital cardiac arrest

who undergo emergency cardiopulmonary bypass and percutaneous coronary intervention.

Circ J 2010;74:77-85.

27. Gaieski DF, Boller M, Becker LB. Emergency cardiopulmonary bypass: a promising 
487 Investigators. Duration of resuscitation efforts and survival after in-hospital cardiac arrest: an

488 observational study. Lancet 2012;380:1473-81.

489 29. Bobrow BJ, Spaite DW, Berg RA, et al. Chest compression-only CPR by lay rescuers

490 and survival from out-of-hospital cardiac arrest. JAMA 2010;304:1447-54.

491 30. Bobrow BJ, Clark LL, Ewy GA, et al. Minimally interrupted cardiac resuscitation by

492 emergency medical services for out-of-hospital cardiac arrest. JAMA 2008;299:1158-65.

493 31. Peberdy MA, Callaway CW, Neumar RW, et al. Part 9: Post-cardiac arrest care: 2010

494 American Heart Association guidelines for cardiopulmonary resuscitation and emergency

495 cardiovascular care. Circulation 2010;122:S768-86.

496

497

498 


\section{$500 \quad$ Legend to Figures}

501 Fig. 1.Patient enrollment flow in both study sites.

502 Finally, 7,759 patients (3,594 from Seoul and 4,163 from Osaka) were enrolled in this study.

503 EMS, emergency medical service; OHCA, out-of-hospital cardiac arrest.

504 Fig. 2.Estimated probability and 95\% confidence intervals of survival with good neurological

505 outcome as a function of the STI using 5-knots restricted cubic spline univariatelogistic

506 regression model.

507 The dark solid line indicates the average probability of survival with good neurological

508 outcome.The red dot lines are cut-off values corresponding to average probability.

509 STI, scene time interval 


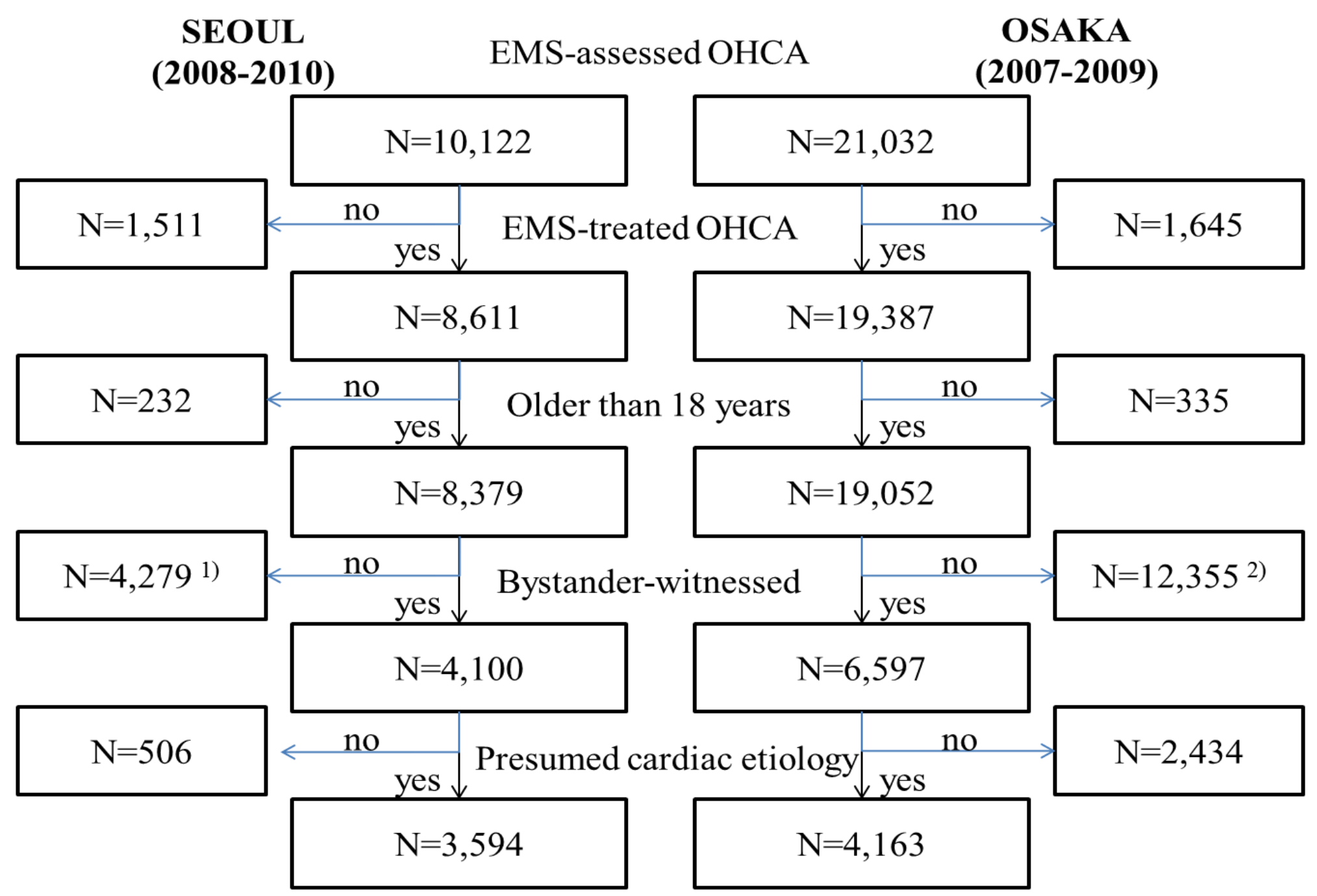

1) 338 witnessed by EMS provider and 3,941 not witnessed by lay person

2) 1,482 witnessed by EMS provider and 10,973 not witnessed by lay person 


\section{Figure 2}

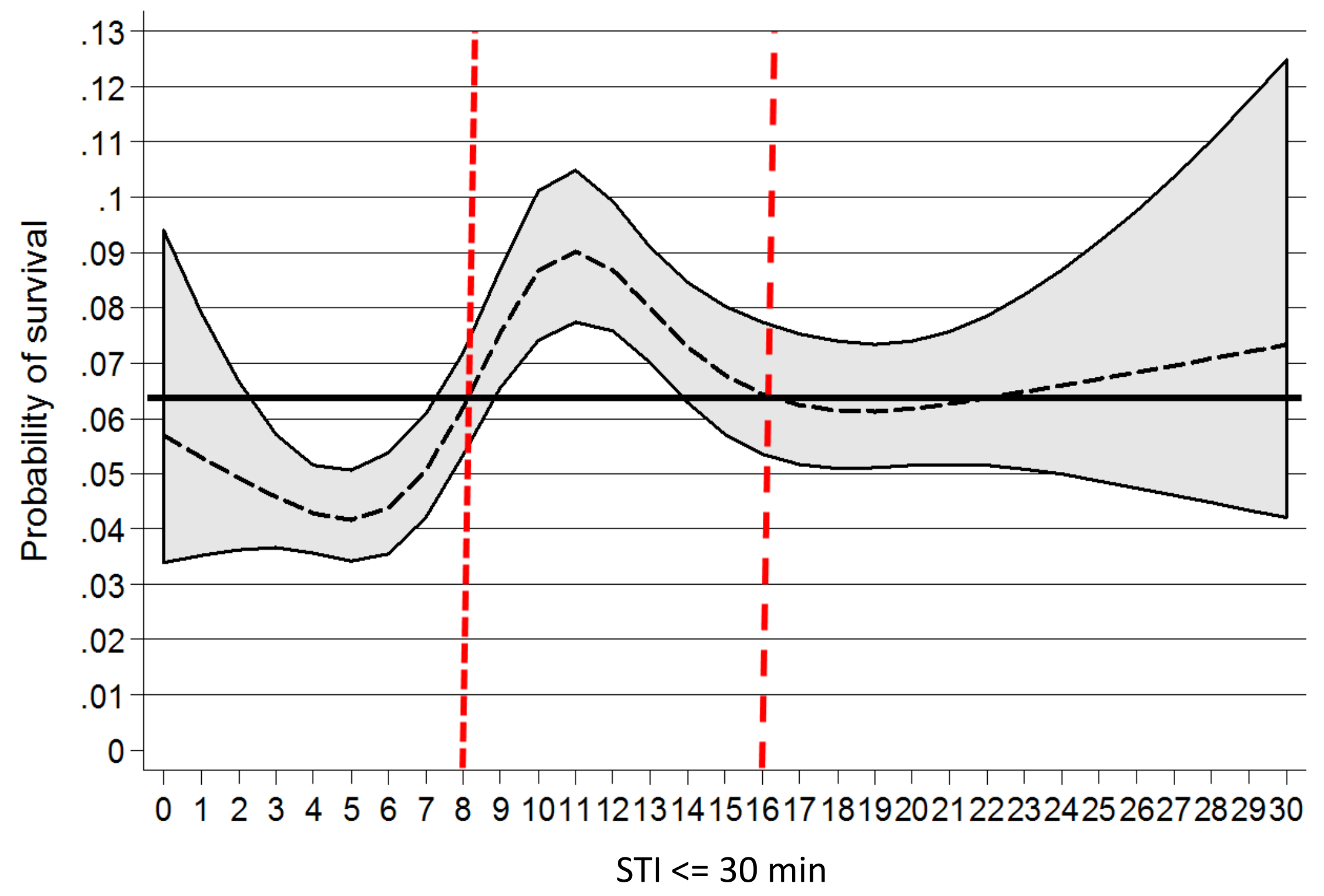


Table 1

Seoul and Osaka demographic findings.

\begin{tabular}{|c|c|c|c|c|c|c|c|}
\hline \multirow[b]{2}{*}{ Number of cases, $n, \%$} & \multicolumn{2}{|c|}{ Total } & \multicolumn{2}{|c|}{ Seoul } & \multicolumn{2}{|c|}{ Osaka } & \multirow[t]{2}{*}{$\underline{p}$} \\
\hline & 7,757 & 100.0 & 3,594 & 100.0 & 4,163 & 100.0 & \\
\hline \multicolumn{8}{|l|}{ Year, $n, \%$} \\
\hline 2007 & 1,317 & 17.0 & 0 & 0.0 & 1,317 & 31.6 & \multirow{4}{*}{ NA } \\
\hline 2008 & 2,508 & 32.3 & 1,108 & 30.8 & 1,400 & 33.6 & \\
\hline 2009 & 2,645 & 34.1 & 1,199 & 33.4 & 1,446 & 34.7 & \\
\hline 2010 & 1,287 & 16.6 & 1,287 & 35.8 & 0 & 0.0 & \\
\hline Female, gender, n, \% & 2,713 & 35.0 & 1,156 & 32.2 & 1,557 & 37.4 & $<0.001$ \\
\hline Age, mean, SD & 68.7 & 16.0 & 64.8 & 15.6 & 72.5 & 15.3 & $<0.001$ \\
\hline \multicolumn{8}{|l|}{ Age group, $n, \%$} \\
\hline 10-19 years & 17 & 0.2 & 10 & 0.3 & 7 & 0.2 & \multirow{8}{*}{$<0.001$} \\
\hline 20-29 years & 126 & 1.6 & 77 & 2.1 & 49 & 1.2 & \\
\hline 30-39 years & 263 & 3.4 & 173 & 4.8 & 90 & 2.2 & \\
\hline 40-49 years & 600 & 7.7 & 397 & 11.0 & 203 & 4.9 & \\
\hline $50-59$ years & 1,041 & 13.4 & 636 & 17.7 & 405 & 9.7 & \\
\hline $60-69$ years & 1,606 & 20.7 & 808 & 22.5 & 798 & 19.2 & \\
\hline 70-79 years & 1,932 & 24.9 & 860 & 23.9 & 1,072 & 25.8 & \\
\hline Older than 80 years & 2,172 & 28.0 & 633 & 17.6 & 1,539 & 37.0 & \\
\hline Public place, $n, \%$ & 2,001 & 25.8 & 677 & 18.8 & 1,324 & 31.8 & $<0.001$ \\
\hline Bystander CPR, $n, \%$ & 2,004 & 25.8 & 257 & 7.2 & 1,747 & 42.0 & $<0.001$ \\
\hline Bystander defibrillation, $n, \%$ & 89 & 1.1 & 0 & 0.0 & 89 & 2.1 & $<0.001$ \\
\hline EMS defibrillation, $n, \%$ & 1,703 & 22.0 & 515 & 14.3 & 1,188 & 28.5 & $<0.001$ \\
\hline \multicolumn{8}{|l|}{ Primary ECG, $n, \%$} \\
\hline VF/pulseless VT & 1,293 & 16.7 & 352 & 9.8 & 941 & 22.6 & \multirow{4}{*}{$<0.001$} \\
\hline PEA & 1,742 & 22.5 & 358 & 10.0 & 1,384 & 33.2 & \\
\hline Asystole & 4,704 & 60.6 & 2,878 & 80.1 & 1,826 & 43.9 & \\
\hline Unknown & 18 & 0.2 & 6 & 0.2 & 12 & 0.3 & \\
\hline \multicolumn{8}{|l|}{ Elapsed time intervals, mean, SD } \\
\hline Response time interval & 7.3 & 3.1 & 7.0 & 3.4 & 7.5 & 2.8 & $<0.001$ \\
\hline Scene time interval & 10.9 & 6.8 & 6.8 & 4.2 & 14.5 & 6.5 & $<0.001$ \\
\hline Transportation time interval & 7.3 & 5.1 & 7.3 & 5.1 & 7.2 & 5.1 & 0.375 \\
\hline Prehospital time interval & 25.4 & 9.8 & 21.2 & 7.9 & 29.0 & 9.8 & $<0.001$ \\
\hline \multicolumn{8}{|l|}{ Outcomes, $n, \%$} \\
\hline Prehospital ROSC & 755 & 9.7 & 110 & 3.1 & 645 & 15.5 & $<0.001$ \\
\hline Survival to admission & 2,608 & 33.6 & 1,033 & 28.7 & 1,575 & 37.8 & $<0.001$ \\
\hline Survival to discharge & 1,008 & 13.0 & 400 & 11.1 & 608 & 14.6 & $<0.001$ \\
\hline Good neurological outcome & 496 & 6.4 & 127 & 3.5 & 369 & 8.9 & $<0.001$ \\
\hline
\end{tabular}

CPR, cardiopulmonary resuscitation; EMS, emergency medical service; ECG, electrocardiography;

ROSC, return of spontaneous circulation; SD, standard deviation; VF, ventricular fibrillation;

VT, ventricular tachycardia; PEA, pulseless electrical activity. 
Table 2

Distribution of risk factors and outcomes by scene time intervals.

\begin{tabular}{|c|c|c|c|c|c|c|c|c|c|}
\hline \multirow[b]{3}{*}{ Total, N, \% } & \multirow{2}{*}{\multicolumn{2}{|c|}{ Total }} & \multirow{2}{*}{\multicolumn{2}{|c|}{$\frac{\text { Short }}{(0=<\text { STI }<8)}$}} & \multirow{2}{*}{\multicolumn{2}{|c|}{$\frac{\text { Intermediate }}{(8=<\mathrm{STI}<16)}$}} & \multirow{2}{*}{\multicolumn{2}{|c|}{$\frac{\text { Long }}{(16=<\mathrm{STI})}$}} & \multirow{3}{*}{$p$} \\
\hline & & & & & & & & & \\
\hline & 7,757 & 100.0 & 2,739 & 100.0 & 3,314 & 100.0 & 1,704 & 100.0 & \\
\hline \multicolumn{10}{|l|}{ Year, N, \% } \\
\hline 2007 & 1,317 & 17.0 & 135 & 4.9 & 712 & 21.5 & 470 & 27.6 & $<0.001$ \\
\hline 2008 & 2,508 & 32.3 & 840 & 30.7 & 1,064 & 32.1 & 604 & 35.4 & \\
\hline 2009 & 2,645 & 34.1 & 919 & 33.6 & 1,138 & 34.3 & 588 & 34.5 & \\
\hline 2010 & 1,287 & 16.6 & 845 & 30.9 & 400 & 12.1 & 42 & 2.5 & \\
\hline City, N, \% & & & & & & & & & $<0.001$ \\
\hline Seoul & 3,594 & 46.3 & 2,353 & 85.9 & 1,119 & 33.8 & 122 & 7.2 & \\
\hline Osaka & 4,163 & 53.7 & 386 & 14.1 & 2,195 & 66.2 & 1,582 & 92.8 & \\
\hline \multicolumn{10}{|l|}{ Gender, N, \% } \\
\hline Male & 5,044 & 65.0 & 1,866 & 68.1 & 2,086 & 62.9 & 1,092 & 64.1 & \\
\hline Female & 2,713 & 35.0 & 873 & 31.9 & 1,228 & 37.1 & 612 & 35.9 & \\
\hline Age group, mean, SD & 68.7 & 16.0 & 65.1 & 16.0 & 70.0 & 15.6 & 72.1 & 15.5 & $<0.001$ \\
\hline Age $<65$ years & 2,741 & 35.3 & 1,217 & 44.4 & 1,075 & 32.4 & 449 & 26.3 & \\
\hline Age $>=65$ years & 5,016 & 64.7 & 1,522 & 55.6 & 2,239 & 67.6 & 1,255 & 73.7 & \\
\hline Place of arrest, N, \% & & & & & & & & & $<0.001$ \\
\hline Public & 2,001 & 25.8 & 726 & 26.5 & 877 & 26.5 & 398 & 23.4 & \\
\hline Private & 5,333 & 68.8 & 1,807 & 66.0 & 2,262 & 68.3 & 1,264 & 74.2 & \\
\hline Unknown & 423 & 5.5 & 206 & 7.5 & 175 & 5.3 & 42 & 2.5 & \\
\hline Bystander CPR, N, \% & & & & & & & & & $<0.001$ \\
\hline No & 5,753 & 74.2 & 2,409 & 88.0 & 2,279 & 68.8 & 1,065 & 62.5 & \\
\hline Yes & 2,004 & 25.8 & 330 & 12.0 & 1,035 & 31.2 & 639 & 37.5 & \\
\hline Bystander defibrillation, N, \% & & & & & & & & & $<0.001$ \\
\hline No & 7,668 & 98.9 & 2,726 & 99.5 & 3,265 & 98.5 & 1,677 & 98.4 & \\
\hline Yes & 89 & 1.1 & 13 & 0.5 & 49 & 1.5 & 27 & 1.6 & \\
\hline EMS defibrillation, $\mathrm{N}, \%$ & & & & & & & & & $<0.001$ \\
\hline No & 6,054 & 78.0 & 2,301 & 84.0 & 2,520 & 76.0 & 1,233 & 72.4 & \\
\hline Yes & 1,703 & 22.0 & 438 & 16.0 & 794 & 24.0 & 471 & 27.6 & \\
\hline Primary ECG, N, \% & & & & & & & & & $<0.001$ \\
\hline VF/pulseless VT & 1,293 & 16.7 & 342 & 12.5 & 601 & 18.1 & 350 & 20.5 & \\
\hline PEA & 1,742 & 22.5 & 381 & 13.9 & 810 & 24.4 & 551 & 32.3 & \\
\hline Asystole & 4,722 & 60.9 & 2,016 & 73.6 & 1,903 & 57.4 & 803 & 47.1 & \\
\hline Unknown & 18 & 0.2 & 8 & 0.3 & 4 & 0.1 & 6 & 0.4 & \\
\hline \multicolumn{10}{|l|}{ Elapsed time intervals, N, \% } \\
\hline Response time, mean, SD & 7.3 & 3.1 & 7.0 & 3.1 & 7.4 & 2.8 & 7.6 & 3.5 & $<0.001$ \\
\hline Transportation time interval, mean, SD & 7.3 & 5.1 & 7.1 & 5.1 & 7.2 & 4.9 & 7.9 & 5.4 & $<0.001$ \\
\hline Prehospital time, mean, SD & 25.4 & 9.8 & 18.7 & 6.5 & 25.7 & 6.5 & 35.5 & 10.5 & $<0.001$ \\
\hline
\end{tabular}

STI, scene time interval from ambulance wheel arrival to departure (unit: minute.); CPR, cardiopulmonary resuscitation; EMS, emergency medical service; ECG, electrocardiography; ROSC, return of spontaneous circulation; SD, standard deviation; VF, ventricular fibrillation; VT, ventricular tachycardia;

PEA, pulseless electrical activity. 
Table 3

Outcomes after OHCA by scene time interval group and city.

\begin{tabular}{|c|c|c|c|c|c|c|c|c|c|c|}
\hline \multirow[t]{2}{*}{ City } & \multirow[t]{2}{*}{ Outcome } & \multicolumn{2}{|c|}{ Total } & \multicolumn{2}{|c|}{$\begin{array}{c}\text { Short } \\
0<=S T I<8 \text { min }\end{array}$} & \multicolumn{2}{|c|}{$\begin{array}{c}\text { Intermediate } \\
8<=\mathrm{STI}<16 \mathrm{~min}\end{array}$} & \multicolumn{2}{|c|}{$\begin{array}{c}\text { Long } \\
16<=\text { STI min }\end{array}$} & \multirow[t]{2}{*}{$P$} \\
\hline & & $\mathrm{N}$ & $\%$ & $\mathrm{~N}$ & $\%$ & $\mathrm{~N}$ & $\%$ & $\mathrm{~N}$ & $\%$ & \\
\hline \multirow[t]{5}{*}{ Total } & Total & 7,757 & & 2,739 & & 3,314 & & 1,704 & & \\
\hline & Preshospital ROSC & 755 & 9.7 & 97 & 3.5 & 374 & 11.3 & 284 & 16.7 & $<0.001$ \\
\hline & Survival to admission & 2,608 & 33.6 & 871 & 31.8 & 1,182 & 35.7 & 555 & 32.6 & 0.004 \\
\hline & Survival to discharge & 1,008 & 13.0 & 359 & 13.1 & 453 & 13.7 & 196 & 11.5 & 0.094 \\
\hline & Good neurological outcome & 496 & 6.4 & 127 & 4.6 & 256 & 7.7 & 113 & 6.6 & $<0.001$ \\
\hline \multirow[t]{5}{*}{ Seoul } & Total & 3,594 & & 2,353 & & 1,119 & & 122 & & \\
\hline & Preshospital ROSC & 110 & 3.1 & 58 & 2.5 & 47 & 4.2 & 5 & 4.1 & 0.017 \\
\hline & Survival to admission & 1,033 & 28.7 & 704 & 29.9 & 303 & 27.1 & 26 & 21.3 & 0.041 \\
\hline & Survival to discharge & 400 & 11.1 & 280 & 11.9 & 107 & 9.6 & 13 & 10.7 & 0.121 \\
\hline & Good neurological outcome & 127 & 3.5 & 83 & 3.5 & 40 & 3.6 & 4 & 3.3 & 0.986 \\
\hline \multirow[t]{5}{*}{ Osaka } & Total & 4,163 & & 386 & & 2,195 & & 1,582 & & \\
\hline & Preshospital ROSC & 645 & 15.5 & 39 & 10.1 & 327 & 14.9 & 279 & 17.6 & $<0.001$ \\
\hline & Survival to admission & 1,575 & 37.8 & 167 & 43.3 & 879 & 40.0 & 529 & 33.4 & $<0.001$ \\
\hline & Survival to discharge & 608 & 14.6 & 79 & 20.5 & 346 & 15.8 & 183 & 11.6 & $<0.001$ \\
\hline & Good neurological outcome & 369 & 8.9 & 44 & 11.4 & 216 & 9.8 & 109 & 6.9 & 0.001 \\
\hline
\end{tabular}

OHCA, out-of-hospital cardiac arrest; STI, scene time interval; ROSC, return of spontaneous circulation. 


\section{Table 4}

Association between scene time intervals and outcomes after OHCA.

\begin{tabular}{|c|c|c|c|c|c|c|c|c|c|}
\hline \multirow{2}{*}{$\begin{array}{l}\text { Outcomes } \\
\text { Scene time interval }\end{array}$} & \multirow{2}{*}{$\frac{\text { Total }}{\mathrm{N}}$} & \multicolumn{2}{|c|}{ Outcome } & \multicolumn{3}{|c|}{ Crude OR } & \multicolumn{3}{|c|}{ Adjusted OR } \\
\hline & & $\mathrm{N}$ & $\%$ & OR & & & $\mathrm{OR}$ & & \\
\hline $0=<\mathrm{STI}<8 \mathrm{~min}$ & 2,739 & 97 & 3.5 & Reference & & & Reference & & \\
\hline $8=<\mathrm{STI}<16 \mathrm{~min}$ & 3,314 & 374 & 11.3 & 3.47 & 2.76 & 4.36 & 2.78 & 2.18 & 3.54 \\
\hline $16=<$ STI $\min$ & 1,704 & 284 & 16.7 & 5.45 & 4.29 & 6.92 & 4.23 & 3.26 & 5.49 \\
\hline Survival to admission, total & 7,757 & 2,608 & 33.6 & & & & & & \\
\hline $16=<$ STI $\min$ & 1,704 & 555 & 32.6 & 1.04 & 0.91 & 1.18 & 0.94 & 0.82 & 1.09 \\
\hline Survival to discharge, total & 7,757 & 1,008 & 13 & & & & & & \\
\hline $0=<\mathrm{STI}<8 \mathrm{~min}$ & 2,739 & 359 & 13.1 & Reference & & & Reference & & \\
\hline $8=<\mathrm{STI}<16 \mathrm{~min}$ & 3,314 & 453 & 13.7 & 1.05 & 0.91 & 1.22 & 0.88 & 0.74 & 1.05 \\
\hline $16=<\mathrm{STI} \min$ & 1,704 & 196 & 11.5 & 0.86 & 0.72 & 1.04 & 0.64 & 0.52 & 0.80 \\
\hline $16=<$ STI min & 1,704 & 113 & 6.6 & 1.46 & 1.13 & 1.90 & 0.92 & 0.68 & 1.26 \\
\hline
\end{tabular}

ROSC, return of spontaneous circulation; STI, scene time interval from wheel arrival to departure; OR, odds ratio; CI, confidence interval.

Adjusted ORs were caluculated by adjusting for city, age, gender, response time interval, transportation time interval, place, bystander cardiopulmonary resuscitation, defibrillation by lay person, defibrillation by emergency medical service (EMS) providers, and primary ECG rhythm in the model. 
Table 5

Association between scene time interval and outcomes considering the effect of interaction terms.

\begin{tabular}{|c|c|c|c|c|c|c|c|c|}
\hline \multirow{2}{*}{ Outcomes } & \multirow{2}{*}{ Effect modifier } & \multicolumn{7}{|c|}{ Scene time interval } \\
\hline & & \multirow[t]{2}{*}{$<8 \min$} & \multicolumn{3}{|c|}{$8-16 \min$} & \multicolumn{3}{|c|}{$>=16 \mathrm{~min}$} \\
\hline \multirow[t]{3}{*}{ Good neurological outcome } & & & AOR & \multicolumn{2}{|c|}{$95 \% \mathrm{CI}$} & $\mathrm{AOR}$ & \multicolumn{2}{|c|}{$95 \% \mathrm{CI}$} \\
\hline & No & Reference & 140 & 108 & 182 & 101 & 074 & \\
\hline & Yes & Reference & 0.20 & 0.05 & 0.92 & 0.09 & 0.02 & 0.44 \\
\hline \multicolumn{9}{|l|}{ Prehospital ROSC } \\
\hline & $\begin{array}{l}\text { Bystander defibrillation } \\
\text { No } \\
\text { Yes }\end{array}$ & $\begin{array}{l}\text { Reference } \\
\text { Reference }\end{array}$ & 2.94 & $\begin{array}{l}2.29 \\
0.09\end{array}$ & $\begin{array}{l}3.78 \\
121\end{array}$ & 4.58 & 3.50 & $\begin{array}{l}5.98 \\
0.98\end{array}$ \\
\hline
\end{tabular}

ROSC, return of spontaneous circulation; AOR, adjusted odds ratio; CI, confidence interval.

AORs were caluculated by constrast estimate for all corresponding interaction variables adjusting for potential confounders in the final model. 